\title{
LA HISTORIOGRAFIA AMBIENTAL EN LA Republica ARgentina
}

\author{
Marina Miraglia \\ Universidad Nacional de General Sarmiento
}

\section{Resumen}

Con motivo de realizarse la III Escuela de Posgraduación de la Sociedad Latinoameriana y del Caribe de Historia Ambiental (SOLCHA) en la Universidad Unievangélica (Goias-Brasil), y en ocasión de participar en la mesa redonda sobre Impactos de la Historiografía Ambiental en la Disciplina Histórica en América Latina, se presenta en este dossier, un trabajo sobre la historiografía ambiental en Argentina, donde se reseñan las principales actividades académicas realizadas en torno al ambiente en las Universidades y Centros de investigación públicos argentinos en los últimos años. Se organiza el artículo por regiones geográficas (Noroeste, Noreste, Cuyo, Patagonia y Pampeana y Metropolitana de Buenos Aires), autores e instituciones y se realiza una reseña de los aportes académicos en la disciplina

Palabras clave: historia ambiental, geografía histórica, historiografía ambiental, Argentina.

\begin{abstract}
On the occasion of the III Graduate School of the Latin American and Caribbean Society of Environmental History (SOLCHA) at the Unievangélica University (Goias-Brazil), and on occasion to participate in the round table on Impacts of Environmental Historiography in Discipline Historically in Latin America, a dossier on environmental historiography in Argentina is presented, where the main academic activities carried out around the environment in the universities and public research centers in Argentina in the last years are reviewed. The article is organized by geographic regions (Northwest, Northeast, Cuyo, Patagonia and Pampeana and Metropolitan Region of Buenos Aires), authors and institutions and a review of the academic contributions in the discipline its made.
\end{abstract}

Key words: environmental history, historical geography, environmental historiography, Argentina.

$E$

I tema ambiental en Argentina comienza a ser incorporado en los documentos institucionles y académicos a finales de la década de 1950, a partir de los trabajos desarrollados por la Comisión Económica para América Latina y el Caribe (CEPAL), organismo de las Naciones Unidas. Así, lentamente comienzan a tratarse estas problemáticas a partir de la revisión y decadencia del modelo extractivista de los recursos naturales, tanto en Argentina como en toda América Latina.

Esta nueva preocupación coincide con la implementación de los modelos desarrollistas, los cuales incorporaron prácticas de fomento de las economías regionales y en ese contexto comenzaron a considerarse tibiamente, los recursos naturales como parte del patrimonio de una región. Entonces los primeros trabajos buscaban realizar diagnósticos, inventarios de los recursos naturales existentes en una región para desarrollarla y así utilizar los recursos naturales como elementos del desarrollo regional desde un punto de vista más economicista.

En esa época, durante la década de 1960, se desarrollaron trabajos a nivel de las ciencias naturales y agropecuarias, como la biología, la incipiente ecología, la agronomía, la climatología, 
la edafología, y luego, estudios de tipo descriptivo, a nivel de inventario, y recién en la década de 1970, cuando surge a nivel mundial la preocupación por los recursos naturales, la población, el crecimiento económico ${ }^{1}$, comienzan a llevarse a cabo los primeros trabajos ambientales que introducen la dimensión territorial que es analizada por la Geografía y una dimensión temporal estudiada por la Historia. Allí surgen los primeros trabajos de Historia Ambiental.

Estas perspectivas multidisciplinarias favorecieron el desarrollo de instituciones que a nivel nacional y regional en Argentina, comenzaron a investigar problemáticas territoriales, tales los casos de los Institutos del Centro Científico Tecnológico (CCT) del CONICET en Mendoza² que se crearon en la década de 1970, el Instituto de Investigaciones Geohistóricas (IIGHI) en la Universidad Nacional del Nordeste ${ }^{3}$ que también se crea a finales de esa década y es Unidad Ejecutora del CONICET y el Centro de Estudios de la Argentina Rural (CEAR) que se crea más recientemente en 2010, en la Universidad Nacional de Quilmes. ${ }^{4}$

Todos ellos comparten como objeto de estudio las problemáticas ambientales, incorporando la dimensión histórico territorial, integrando disciplinas y herramientas interdisciplinarias tendientes a adquirir un entendimiento más profundo, complementando la descripción con la explicación de los fenómenos que se producen a lo largo de determinados períodos históricos.

Es dentro de estos centros e institutos académico universitarios donde se han desarrollado la mayor parte de los estudios ambientales en la República Argentina, presentando en este artículo una síntesis de los trabajos más recientes sobre la temática, reflejando no sólo la problemática ambiental, sino también los ejes disciplinares que más han trabajado.

Los documentos aquí analizados comprenden el territorio argentino dentro de un período temporal secular para algunos casos y para otros decádico, mostrando la amplitud temática, conceptual, territorial y temporal que se encuentra en la historiografía ambiental en nuestro país.

\section{Las disciplinas científicas y los investigadores que impactan la historiografía ambiental en Argentina}

En Historia, se destacan los trabajos de María del Rosario Prieto (Instituto Argentino de Nivología, Glaciología y Ciencias Ambientales (IANIGLA) dependiente del Centro Científico Tecnológico de la provincia de Mendoza, la Universidad de Cuyo y la provincia de Mendoza), Adrián Zarrilli, Gabriel Garnero y Noemí María Girbal Blacha (CEAR/Universidad Nacional de Quilmes) y Brisa Varela (Universidad Nacional de Luján/Universidad de Buenos Aires/Universidad Nacional de San Martín).

En Geografía, muchos autores se encuentran trabajando en temas de historia ambiental y geografía histórica como son los casos de Elena Abraham (Instituto Argentino de Investigaciones de Zonas Aridas (IADIZA) dependiente del Centro Científico Tecnológico de la provincia de Men-

\footnotetext{
${ }^{1}$ Descriptos a partir de los trabajos del Club de Roma, la Reunión de Estocolmo el informe Bruntland, manifestando las preocupaciones por los problemas ambientales, en Europa sobre todo en Alemania por los problemas de la lluvia ácida, los residuos en todo Europa, la contaminación nuclear, principalmente.

2 http://www.mendoza-conicet.gob.ar/portal/

3 http://www.iighi-conicet.gob.ar/

4 http://cear.blog.unq.edu.ar/
} 
doza, la Universidad de Cuyo y la provincia de Mendoza), Carlos Reboratti (Universidad de Buenos Aires), Rodolfo Richard Jorba (Instituto de Ciencias Humanas, Sociales y Ambientales (INCIHUSA) dependiente del Centro Científico Tecnológico de la provincia de Mendoza, la Universidad de Cuyo y la provincia de Mendoza), Roberto Herrera y Alfredo Bolsi (Universidad Nacional de Cuyo) y Enrique Bruniard (UNNE) y más recientemente, en los últimos 15 años, Cristina Carballo (Universidad Nacional de Luján y Universidad Nacional de Quilmes), Hortensia Castro (Universidad de Buenos Aires); Marina Miraglia (Universidad Nacional de General Sarmiento y Universidad Nacional de Quilmes) y Facundo Rojas (IANIGLA)

En las disciplinas naturales como Agronomía, Biología, Ciencias Naturales, Ecología se incorporan en esta reseña algunos trabajos de Jorge Morello, Jorge Adámoli, José Dadon y Dina Foguelman (UBA), y Pablo Eugenio Villagra, Silvina Stutz y Marcela Tonello (IANIGLA, Centro Científico Tecnológico de la provincia de Mendoza, la Universidad de Cuyo y la provincia de Mendoza).

En temáticas vinculadas con la Sociología, se encuentran los trabajos pioneros de Héctor Alimonda (Consejo Latinoamericano de Ciencias Sociales -CLACSO), en Economía, los trabajos y libros de Antonio Brailovsky (Universidad de Buenos Aires y Universidad de Belgrano) y en la reciente disciplina de la Gestión ambiental, los avances de Lucrecia Wagner (IANIGLA).

\section{Las instituciones que realizan investigaciones ambientales en la Argentina vincula- das con el Consejo Nacional de Investigaciones Científicas y Técnicas (CONICET)}

Como se mencionara anteriormente, son tres los centros con mayor trayectoria en la temática ambiental en la Argentina, ellos son el Centro de Estudios de la Argentina Rural (CEAR) en la Universidad Nacional de Quilmes (UNQ) creado en el año 2010, se propone estudiar los diversos aspectos de la Argentina rural, principalmente asociados a las continuidades y cambios. El Centro Científico Tecnológico (CCT) depende del CONICET, de la Universidad Nacional de Cuyo y la provincia de Mendoza "promueve la transferencia de conocimiento y tecnología a la sociedad, mediante convenios, acuerdos y asesorías a empresas, organizaciones sociales y gubernamentales y hace llegar su experiencia en investigación y desarrollo a los diferentes sectores socioeconómicos regionales, nacionales e internacionales."

El Instituto de Investigaciones Geohistóricas (IIGHI), dependiente del CONICET, sito en la Universidad Nacional del Nordeste (UNNE), con sede actual en la ciudad de Resistencia (provincia de Chaco), tiene como principal objetivo la producción y promoción de conocimiento científico en temas sociales y culturales, en la región Nordeste de Argentina y países limítrofes y busca contribuir a la formación de profesionales calificados para el estudio de la problemática histórico geográfica. Desarrolla actividades de extensión, servicios académicos, docentes y de divulgación y contribuye en la formación de un repositorio cartográfico, bibliográfico y documental sobre el Nordeste, Paraguay y sur de Brasil. 


\section{Estudios de caso}

A fin de ejemplificar la diversidad de enfoques disciplinares y ejes temáticos, se organizan los trabajos por regiones geográficas, tales como Noreste (NEA), Noroeste (NOA), Cuyo, Patagonia y Pampeana y Metropolitana de Buenos Aires (Figura 1). ${ }^{5}$

En primer lugar, se presentan algunos ejemplos seleccionados para la Argentina sobre aspectos socioeconómicos, ambientales en general, variabilidad climática, aportes antropológicos y ecológicos, históricos y geopolíticos.

En el caso del NOA (provincias de Salta, Jujuy, Tucumán, Santiago del Estero, Catamarca y La Rioja), se seleccionaron tres trabajos realizados por investigadores de la región, sobre microfauna y condiciones ambientales, paleoambientales y arqueológicas, así como la relación de la palinología con las condiciones climáticas locales y regionales y los deslizamientos de tierras (eventos catastróficos para la población del NOA).

En la región de Cuyo (provincias de San Juan, Mendoza y San Luis) se hizo una selección de algunos trabajos de geografía histórica e historia ambiental, principalmente urbana y sobre el uso de los recursos naturales, reconstrucción de la vegetación histórica mediante el uso de fuentes documentales, las variaciones climáticas y la evocación del ciclo del oro.

En la región de NEA (Corrientes, Misiones, Chaco y Formosa), se presentan reseñas de trabajos variados, desde un diagnóstico ambiental realizado sobre la ecorregión del chaco seco, pasando por otro trabajo realizado sobre la base ecológica y el tipo de producción económica en Chaco, y finalmente el aporte de la cartografía histórica en Misiones para la reconstrucción ambiental.

Para el caso de la Patagonia argentina (Neuquén, Río Negro, Chubut, Santa Cruz y Tierra del Fuego) se reseña un trabajo sobre Deterioro ambiental y micromamíferos durante el Holoceno combinando historia ambiental, paleoecología, climatología y geología, y un trabajo de historia ambiental donde se presenta la reconstrucción histórica de las relaciones entre la sociedad y los recursos naturales.

Finalmente, para la región pampeana (Buenos Aires, Santa Fé, Córdoba, Entre Ríos y La Pampa) y metropolitana de Buenos Aires (40 municipios y la Ciudad Autónoma de Buenos Aires) se presentan trabajos de tipo metodológicos, la integración de lineamientos teóricos en la Historia Ambiental, el análisis de las fluctuaciones de agua y los proyectos hídricos en las cuencas del noroeste de Córdoba, un análisis estratégico militar, un estudio del litoral bonaerense y la incorporación de sus tierras al turismo y un análisis histórico hidrológico de cuerpos lacunares.

A continuación se reseñan los trabajos mencionados.

\footnotetext{
${ }^{5}$ Se adopta un criterio operativo basado en una adecuación personal realizada sobre la clasificación de regiones productivas en Argentina, utilizada por autores institucionales (Instituto Nacional de Estadísticas y Censos (2010), Ministerio de Turismo de la Nación (2014), Ministerio de Planificación Federal, Inversión Pública y Servicios (2010), entre los más destacados).
} 


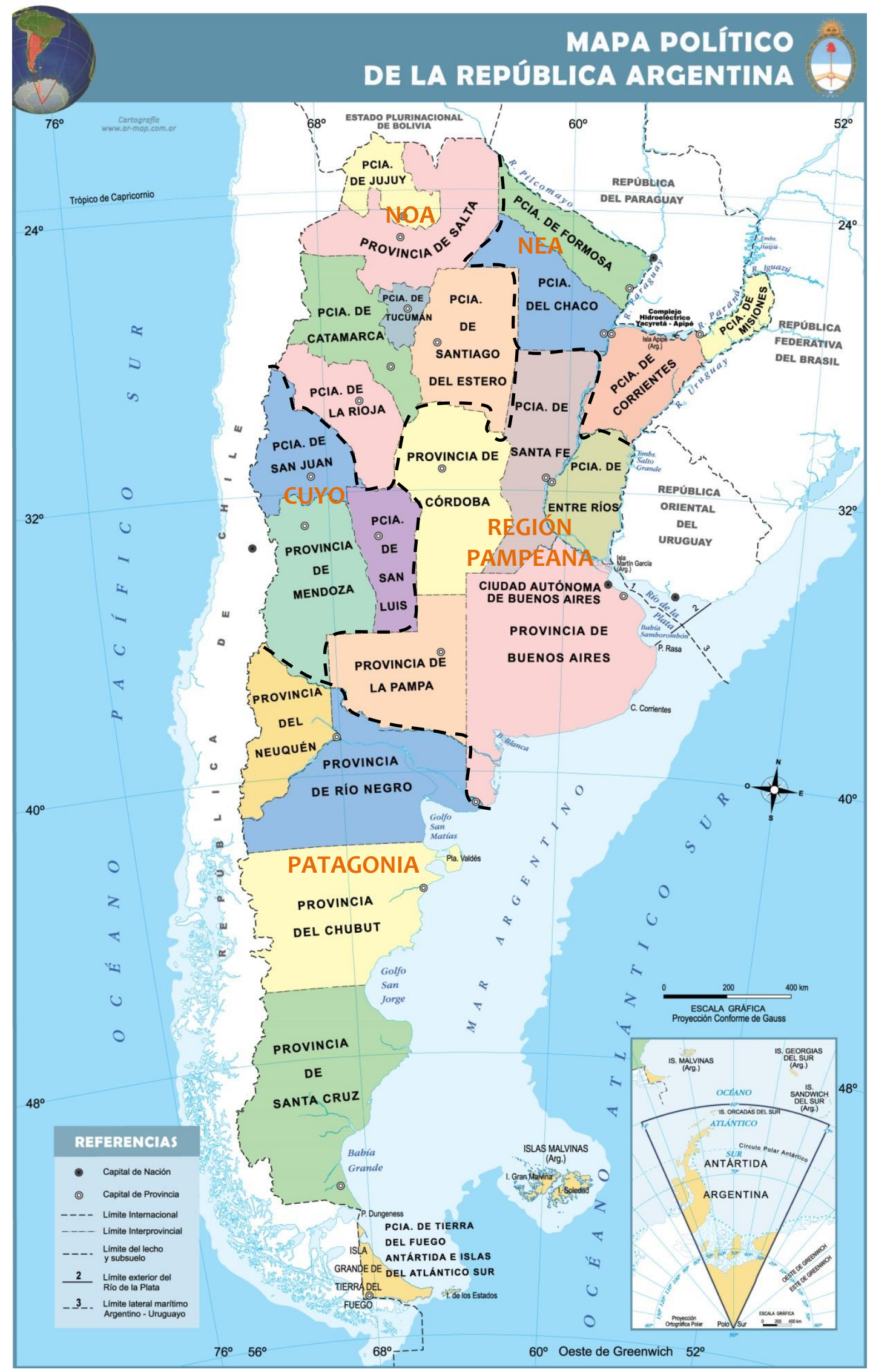

Figura 1. Regiones geográficas operativas de la Argentina (adaptado). Fuente: http://mapoteca.educ.ar/.files/index.html.1.27.html. 


\section{Argentina}

En el trabajo sobre "Pobreza y políticas urbano-ambientales en Argentina", publicado por la CEPAL en la serie Medio Ambiente y Desarrollo, la autora hace una aproximación conceptual y metodológica sobre la pobreza, exclusión y vulnerabilidad y la producción de la ciudad y segregación socio territorial aplicando estos conceptos y metodología en el caso de la Argentina y la RMBA. Otro aspecto considerado son las Instituciones y políticas urbano-ambientales y la planificación urbana y su relación con la normativa ambiental: instituciones y políticas en el nivel nacional, en la ciudad autónoma de Buenos Aires, en la Provincia de Buenos Aires y el nivel municipal y el caso particular de la regulación de la localización industrial. Las políticas de tierra y sus consecuencias sobre el ambiente urbano de la pobreza son otros ítems analizados en su trabajo (CLICHEVSKY, 2002).

Desde el punto de vista de la relación entre la Antropología y la Historia Ambiental, se presentan los potenciales aportes de la Antropología Social, la Ecología Política al campo de la Historia Ambiental. Las autoras proponen a la Etnografía como una buena herramienta metodológica para los estudios de Historia Ambiental. Por último, incorporan algunas investigaciones que se desarrollan en Argentina desde la Historia Ambiental y la Antropología, y cómo esta última puede contribuir a los estudios sobre conflictos y relaciones socio-ambientales teniendo en cuenta procesos de larga duración (SALDI, 2013).

En el libro "Historia ecológica de Iberoamérica: De la independencia a la globalización", Antonio Brailovsky se refiere a los procesos y condiciones ambientales de los países latinoamericanos durante los siglos XIX y XX y perspectivas a futuro. Analiza las condiciones ambientales durante las guerras de la Independencia, detalla la relación con los recursos naturales y el ambiente durante el período de europeización (desde mediados del siglo XIX hasta los primeros años del $X X$ ), donde las migraciones internacionales y la extensión de las líneas ferroviarias son algunas de las piezas claves de este proceso. La etapa siguiente, de sustitución de importaciones, incluye nuevas formas de relación entre la sociedad y el Estado y de estos con los recursos naturales. Las migraciones internas, el rápido crecimiento de las ciudades y la contaminación industrial caracterizan a esta etapa. La última etapa, de globalización, se desarrolla a partir de los procesos de privatizaciones y desindustrialización iniciados a fines del siglo $X X$ con un marcado deterioro de las condiciones de vida de grandes sectores de la población (BRAILOVSKY, 2008).

En el documento "Argentina, tierra de promisión. Una interpretación historiográfica de las relaciones entre la historia rural y la historia ambiental", el autor analiza cómo las diferentes tradiciones que constituyeron el corpus principal de la historia rural argentina interpretaron, incorporaron o percibieron las relaciones sociedad y ambiente en sus explicaciones acerca del desarrollo rural argentino; cuales fueron sus progresos, tendencias y comprensiones complejas (ZARRILLI, 2014).

En este libro, "Historia, política y gestión ambiental", el autor presenta una compilación de trabajos como un aporte en los estudios socioambientales, desde diferentes abordajes de las cuestiones planteadas. El eje del libro está centrado en torno a aportes que se centran en las interrelaciones entre sociedad y naturaleza. Es un abordaje heterogéneo en cuanto a marcos de referencia ideológico, con un amplio espectro temático y un encuentro interdisciplinario. Plantea la 
necesidad de acrecentar la participación de las ciencias sociales en el análisis de estas problemáticas. Una perspectiva solamente visualizada desde las ciencias naturales impide conocer en toda su diversidad y complejidad la cuestión centrada en la construcción social del ambiente (ZARRILLI, 2012).

En el trabajo "Apuntes para una historia ecológico-política de la explotación de los hidrocarburos en Argentina", se revisa la historia petrolera argentina desde el campo de la ecología política a través del análisis de tres grandes etapas: la estatal, la privada y la mixta. En cada de ellas se analizó la articulación entre régimen de acumulación, forma de Estado y apropiación material y simbólica de los bienes de la naturaleza, en este caso el petróleo y el gas. Previamente, el autor incorpora algunas discusiones teóricas y metodológicas en torno a las contribuciones que el marxismo ecológico y la economía política argentina pueden hacer a la ecología política (SABBATELLA, 2013).

\section{Región del Noroeste Argentino (NOA)}

Desde el punto de vista arqueológico, hay un estudio desarrollado sobre la fauna en Piedra Horadada 2, provincia de Catamarca donde se presenta el análisis de un conjunto arqueológico y faunístico, integrando sus aspectos taxonómicos, tafonómicos y económicos, así como la consideración de su distribución espacial, estratigráfica y contextos asociados. Las óptimas condiciones ambientales de conservación del material permitieron abordar un registro variado, conformado por restos óseos, fibras, insectos, plumas, etc. En conjunto con las características del emplazamiento se pudieron reconstruír las prácticas sociales en el marco general de la dinámica ocupacional de grupos pastoriles (URQUIZA et al., 2013).

En otro sentido, se presenta un trabajo de Historia ambiental de la Laguna Seca, Tartagal, Salta, con los resultados de una investigación interdisciplinaria sobre la historia del paisaje, ya incorporando en el título el concepto de historia ambiental, para un período de aproximadamente 53 años (1959-2012). Se trabajó con los sedimentos de la laguna utilizando herramientas de la geoquímica, mineralogía y palinología. Los resultados de la aplicación estas herramientas fueron consistentes con la precipitación anual en la localidad de Tartagal en las últimas décadas, al mismo tiempo que reflejan una alta correlación con las inundaciones y deslizamientos de tierra en la región montañosa (FIERRO, 2016).

Por otro lado, en el trabajo de tesis de doctorado titulado: "Arqueología y paleoambiente: dinámica social y cambio ambiental en sociedades complejas de la Puna argentina" se realizó un análisis paleoambiental de Antofagasta de la Sierra en la provincia de Catamarca, integrando distintas disciplinas como la biología, la geología, la geografía y la antropología, para entender mejor los procesos de transformación e interrelación entre el ambiente y la sociedad andina durante los últimos 6000 años. Dice el autor que "Resulta vital conocer la historia ambiental de una región si pretendemos comprender de forma holística la dinámica social, puesto que los procesos de complejidad social fueron y son productos de la combinación de las condiciones sociales y ambientales dadas en un momento temporal y espacial especifico. Por lo tanto, el análisis de esta complejidad implica estudios de amplios rangos temporales y espaciales para poder entender su emer- 
gencia y su proceso, teniendo siempre en cuenta tanto la dinámica cultural como la ambiental en las que se desarrolla" (GRANA, 2014).

\section{Región de Cuyo}

En el trabajo sobre la utilización de documentos históricos en la reconstrucción de la vegetación de la Llanura de la Travesía (Argentina) a principios del siglo XIX, se buscó reconstruir las condiciones ambientales de dicho ambiente, combinando la información de diversas fuentes documentales. Este análisis reveló un gradiente similar al actual, donde se pudo verificar un aumento de la complejidad de la vegetación en el sentido oeste-este. Se comprueba la validez de la utilización de información documental para reconstruir la vegetación del pasado y detectar cambios ambientales (PRIETO, 2003).

En el trabajo realizado sobre el desierto de Lavalle en Mendoza, se analizan las posibles relaciones existentes entre las dinámicas de uso de los recursos naturales y las estrategias de localización de las unidades domésticas en el territorio, dentro de espacios rurales de desierto que poseen una oferta ambiental limitada. Se analiza un estudio de caso y, dentro de él, se procede con las técnicas cualitativas de entrevista en profundidad y observación participante. Así, la información resultante sugiere que las limitaciones que impone el medio ambiente son sobrellevadas mediante una estrategia de localización relacionada con el proceso de producción, que maximiza el acceso de determinados recursos naturales y que "ordena" la competencia por ellos (TORRES, 2008).

En el artículo denominado "Procesos socioeconómicos y territoriales en el uso de los recursos forestales en Mendoza desde fines de siglo XIX hasta mediados del XX", el autor describe e interpreta los procesos de uso de los bosques de algarrobo en Mendoza entre 1900 y 1942 , asociados al proceso de modernización capitalista y se analiza el rol que los ferrocarriles cumplieron en torno al aprovechamiento intensivo del recurso forestal, como así la satisfacción de la demanda de madera de la industria vitivinícola en auge y de una población urbana en franco ascenso, justamente como se desprende de lo expresado en el presente trabajo, realizado a partir de estadísticas ferroviarias y fuentes históricas. Se reconstruyen algunos aspectos de la historia ambiental regional para comprender los procesos de construcción de estos espacios (ROJAS, 2009).

El trabajo sobre "Procesos sociales y ambientales en el sector urbano de Mendoza entre los siglos XV-XVIII: Arqueología urbana e historia ambiental" contempla un análisis histórico de la ciudad de Mendoza entre los siglos XV y XVIII. Se basó en información estrictamente procedente de la ciudad e inmediaciones y se partió de un modelo elaborado a partir de dos dimensiones: diacrónica procesual y sincrónica funcional. En el primer caso se postula la integración de la ciudad a un proceso complejo de interacciones entre poblaciones nativas y europeas, previas a la fundación urbana, lo que significa desarrollos divergentes, dados entre la efectividad adaptativa y la exploración respectivamente. Presenta además una síntesis sobre las condiciones de emplazamiento de la ciudad y realiza un relevamiento historiográfico donde se reevaluará la documentación y se contrastará el modelo sumando para ello evidencias materiales trabajadas desde la arqueología urbana y documentales desde la historia ambiental (CHIAVAZZA, 2010). 
En el artículo "Recursos, sociedade y ambiente: el ciclo del oro em la organización ambiental del território serrano de la Provincia de San Luis del siglo XVIII", la autora realiza la reconstrucción ambiental del siglo de oro en San Luis (Argentina), introduciendo en la relación entre las actividades económicas, la configuración ambiental del territorio y la sociedad. Se proponen cinco aspectos centrales: las fases del ciclo minero y sus relaciones con los movimientos de población; los problemas medioambientales; el alcance y los límites de la tecnología disponible; la valorización económica y política a partir del Estado; y las formas de ocupación del área (VARELA, 2001).

\section{Región del Noreste Argentino (NEA)}

En el texto "Situación ambiental de la Ecorregión del Chaco seco" (TORRELLA, 2005), el autor realiza un diagnóstico ambiental sobre esta ecorregión, una vasta llanura sedimentaria, modelada esencialmente por la acción de los ríos que la atraviesan en sentido noroeste-sudeste, principalmente el Juramento-Salado, el Bermejo y el Pilcomayo. Sus altas cuencas se encuentran fuera de la región, en la cordillera, desde donde transportan una gran cantidad de sedimentos que forman albardones a los costados del cauce 0 , como ocurre con frecuencia, colmatan los cauces y dan origen a la divagación de los ríos.

Estas divagaciones forman abanicos (o paleo abanicos) fluviales, caracterizados por la presencia de paleoalbardones con una cobertura vegetal (muchas veces en desequilibrio con el régimen hídrico actual) y paleocauces de suelos arenosos, generalmente cubiertos por pastizales que atraviesan la matriz boscosa de la región. Estos paleocauces fueron, a fines del siglo XIX y a comienzos del XX, las vías de acceso de los colonos ganaderos que se establecían en la región. El fuego es otro importante factor que actúa como modelador del paisaje a nivel regional, es también manipulado por el hombre, sin embargo, cuando es practicado en forma inadecuada, impacta negativamente el ambiente.

En el trabajo titulado "Transformación ecológica y precariedad económica en una economía marginal. El Gran Chaco argentino, 1890-1950" (ZARRILLI, 2000), el autor realiza un análisis sobre su base ecológica y de sus recursos naturales, mientras que el tipo y grado de desarrollo afectan directamente esa base ambiental. El objetivo esencial de la Historia Ecológica se centra en el conocimiento de la lógica económica, de las normas éticas y culturales propias de cada forma histórica de producción, ya que al influir en las prácticas de los agentes sociales en relación al medio, determinan el menor o el mayor grado de sostenibilidad de la producción. La destrucción ecológica, la sobreexplotación de los recursos naturales y la degradación ambiental han caracterizado los procesos de incorporación de las tierras boscosas a la economía capitalista. Los recursos ambientales han sido una condición, pero también una externalidad y un costo del proceso de producción de ganancias y excedentes económicos. La riqueza forestal y el aprovechamiento de las maderas más buscadas, se constituyó a mediados de la década de 1890, en el atractivo económico más importante para la penetración en la zona del Gran Chaco Argentino.

En "Aportes de la cartografía histórica para una historia ambiental en Misiones, Argentina (1880-1980)", Gallero reflexiona sobre el aporte heurístico de la cartografía para analizar la interrelación entre la naturaleza y la sociedad a través del tiempo y los cambios y permanencias en el 
medioambiente que se estudia, en este caso la provincia de Misiones, Argentina. En este sentido, la historia ambiental, como "saber ambiental" se proyecta "hacia el infinito de lo impensado -lo por pensar - reconstituyendo identidades en la reapropiación del mundo" (LEFF, 2009, p. 246). Un saber que busca descubrir la complejidad de la transformación del medioambiente de la provincia de Misiones, y que trata de comprender a través de diferentes mapas las huellas que dejó la "territorialización del estado" y descubrir cómo la cartografía fue mostrando la "intersección"entre la sociedad y la naturaleza. A fines expositivos se dividió el trabajo en tres grandes períodos, organizados por las características principales que definieron el proceso de poblamiento desde que se creó el Territorio Nacional (1881) hasta la última edición del "Gran Atlas de Misiones" (2013). El primer período (1881-1920) se caracteriza por la colonización promovida por el estado y la ocupación espontánea; el segundo por el poblamiento a partir de la iniciativa privada (1920-1953); y el tercero (1953-2013) por la consolidación y organización de una provincia. La selección de la cartografía es el resultado de un largo proceso de búsqueda en el trabajo de campo de mi tesis doctoral (GALLERO, 2009).

\section{Región Patagónica}

En esta región se encuentran gran cantidad de trabajos en todas las disciplinas y ejes temáticos, un ejemplo de ello es el trabajo sobre Deterioro ambiental y micromamíferos durante el Holoceno en el nordeste de la estepa patagónica (PARDIÑAS, 2000) donde estudiaron la composición taxonómica de micromamíferos del paraje Lle cul, en el valle del río Chubut, con una antigüedad entre 1760 a 2000 años según la datación radiocarbónica. En este trabajo se realizó un análisis que demostró que la expansión de los agroecosistemas habría favorecido la dispersión y abundancia de una especie (Calomys) en detrimento de otros micromamíferos.

Hay un trabajo asociado a la reconstrucción de la historia ambiental de las tierras secas chubutenses (EJARQUE, 2013) que consideró los modos en que se ha ido modificando la relación sociedad-naturaleza en diferentes períodos. Desde los orígenes del poblamiento de este espacio hasta la complejidad de la situación actual, en esta historia se han seleccionado algunas variables claves para comprender la imbricada relación que han tenido la sociedad y la naturaleza, a través de la ganadería ovina para la producción de lanas. Sin duda, la crítica situación de los recursos naturales en la región encuentra sus bases en la forma en que se desarrolló esta relación históricamente. Según la autora, esta reconstrucción histórica se realizó a partir del análisis de fuentes documentales (principalmente de diarios y revistas de la región) y la utilización de investigaciones historiográficas específicas del área de estudio y de la Patagonia.

\section{Región pampeana y metropolitana de Buenos Aires}

Desde un eje paleoclimatico, Stutz (2014) presenta un estudio con el objetivo de reconstruir la historia evolutiva de cinco lagos someros de la llanura Pampeana (Argentina) desde el Holoceno medio, y así realizar inferencias paleoclimáticas para esta región. Durante el Holoceno medio y 
por largos períodos (alrededor de 6.500 años) la estabilidad del paisaje se mantuvo debido a las condiciones climáticas más secas que las actuales, en las que predominaron fluctuaciones de sequías e inundaciones y/o condiciones de alta evaporación.

Otro aspecto considerado es la historia hidrológica, como el trabajo denominado "Cambios ambientales de épocas históricas en la pampa bonaerense en base a ostrácodos: historia hidrológica de la laguna de Chascomús" (LAPRIDA, 2009). Aquí se reconstruye la evolución para los últimos 500 años. La cronología de esta evolución es comparada con los registros históricos recopilados para el área. Estos ciclos tuvieron lugar bajo escenarios climáticos diferentes respecto de la disponibilidad de agua en el sistema.

Desde el punto de vista turístico, el trabajo "Historia ambiental y turismo en la costa bonaerense: de playas, vacaciones y ecologia" (DADON, 2005) presenta las características ambientales del litoral bonaerense donde se instalaron en el último siglo las principales localidades turísticas de esta región. En el siglo XIX, las costas medanosas eran consideradas estériles, improductivas e inadecuadas para ser habitadas. En contraposición, a fines del siglo XX el valor de esos terrenos superaba al de las zonas agrícolas. Su incorporación a la economía nacional fue resultado de importantes cambios sociales y culturales, que a su vez generaron profundas transformaciones ambientales. En menos de un siglo, las playas antaño solitarias pasaron a ser asiento de nuevas actividades, nuevas ciudades, nuevos usos sociales e inclusive nuevas especies.

Hay trabajos de tipo estratégico militares (DESCHAMPS, 2007) como el denominado "Aspectos ambientales en torno al primer fuerte de la frontera sur de Buenos Aires: "El Zanjón" 17451779", que tiene como objetivo reconstruir los datos ambientales y climáticos de la zona en esa época cuando en la segunda mitad del siglo XVIII el Cabildo de Buenos Aires decide comenzar la extensión de la frontera sur rodeando a la incipiente población con una serie de fortines que protegieran a sus habitantes e impidieran el robo de ganado.

También hay trabajos de tipo teórico y metodológico (MIRAGLIA, 2016), donde se analiza el territorio como sistema complejo. La dinámica territorial está compuesta por variados elementos interdefinibles entre si y múltiples interrelaciones. Esos elementos son variables físico ambientales, socio económicas, así como factores climáticos, políticos y culturales, los que, al desarrollarse en un proceso geohistórico, requieren de la identificación, exploración y análisis de sus dimensiones espacio temporales, a través de la Geografía y la Historia, y particularmente, la Geografía Histórica y la Historia Ambiental. En este trabajo se presentan algunas aplicaciones con el objetivo de identificar la reconstrucción de procesos socioeconómicos y ambientales pretéritos y su ubicación geográfica como base cartográfica diagnóstica en general y como insumo para el análisis espacial del desarrollo histórico de grandes biomas, o de las grandes regiones metropolitanas, y su posterior planificación y ordenamiento territorial.

Los viajeros y científicos recorrieron vastos territorios de los nuevos mundos con el claro propósito de inventariar la gran riqueza faunística y florística para su aprovechamiento por las grandes potencias imperialistas europeas de los siglos XVIII y XIX (MIRAGLIA, 2017). Las sociedades científicas europeas en sintonía con sus gobiernos enviaron a los académicos y científicos aventureros a recopilar información de los nuevos territorios donde invertir sus esfuerzos para extraer los recursos naturales necesarios para la reproducción de la vida en sus sociedades. En la República Argentina, los científicos y viajeros europeos inventariaron con gran detalle las condici- 
ones físico naturales y antropológicas de nuestro territorio. En este artículo se utilizaron algunos de ellos como fuentes de datos para representar la reconstrucción histórica de parte del territorio bonaerense en el siglo XIX.

Vinculado a la problemática característica de la región pampeana como son las inundaciones y sequías, las fluctuaciones estacionales de agua y el origen de los grandes proyectos hídricos en las cuencas del noroeste de Córdoba-Argentina (1880-1925), tenemos el trabajo de Garnero (2017). En los albores de la configuración del modelo agroexportador en Argentina, con la consolidación del estado y la conformación de cuadros técnicos modernos, la elite dirigente provincial fue reafirmando su discurso sobre la "inadecuación" de las características naturales de los valles del noroeste provincial. A su vez, queremos describir como diferentes agentes sociales visualizaron esas amenazas y progresivamente intervinieron sobre las corrientes.

\section{Conclusiones}

En este trabajo se presentaron algunos ejemplos que se constituyen en casos pertenecientes a la historiografía ambiental en Argentina y permiten mostrar los variados intereses y perspectivas disciplinarias que se aplican en estos estudios, realizados en todo el país, a escalas nacional, provincial, regional y local, tendientes en todos los casos a profundizar el conocimiento del ambiente en sí, en sus componentes, en su condición de sistema complejo, en sus relaciones con la sociedad y con las formas de producción predominantes.

Este articulo, es un resumen temático y autoral, que busca constituirse en un aporte al conocimiento de la historiografía ambiental nacional y regional, y que se pueda profundizar posteriormente en otros proyectos. 
Referencias

BRAILOVSKY, A.E. Historia ecológica de Iberoamérica II: De la independencia a la globalización, Buenos Aires: Capital Intelectual, 2008.

CHIAVAZZA, H. Procesos sociales y ambientales en el sector urbano de Mendoza entre los siglos XV-XVIII: Arqueología urbana e historia ambiental. Comechingonia virtual, Revista Electrónica de Arqueología v. IV, n. 2, p. 227-253, 2010.

CLICHEVSKY, N. Pobreza y políticas urbano-ambientales en Argentina. Serie Medio Ambiente y Desarrollo v. 49. CEPAL - ECLAC, Santiago de Chile, 2002.

DADON, J.R. Historia ambiental y turismo en la costa bonaerense: de playas, vacaciones y ecología. Reproducido de: Todo es Historia, n. 450, p. 54-62, 2005.

DESCHAMPS, J.R. Y E.P. TONNI. Aspectos ambientales en torno al primer fuerte de la frontera sur de Buenos Aires: "El Zanjón" 1745-1779. Documento de Trabajo n. 175, Universidad de Belgrano, Facultad de Ciencias Agrarias, Departamento de Investigaciones, 2007.

EJARQUE, M. Reconstruyendo la historia ambiental de las tierras secas chubutenses (Argentina). Zonas Áridas v. 15, n. 1, p. 169-187, 2013.

FIERRO, P.T., J.J. KULEMEYER, L.C. LUPO, S. GIRALT. Historia ambiental de la Laguna Seca, Tartagal, Salta, Noroeste argentino. Revista Brasileira de Paleontologia v. 19, n. 2, p. 325340, 2016.

GALLERO, M.C. Aportes de la cartografía histórica para una historia ambiental en Misiones, Argentina (1880-1980). Revista História: Debates e Tendências v. 16, n. 1, p. 113-134, 2016.

GARNERO, G. Crecientes y sequías: las fluctuaciones estacionales de agua y el origen de los grandes proyectos hídricos en las cuencas del noroeste de Córdoba-Argentina (1880-1925). Temas americanistas n. 38, p. 107-129, 2017.

GRANA, L. Arqueología y paleoambiente: dinámica social y cambio ambiental en sociedades complejas de la Puna argentina. Arqueología v. 20, n. 2, p. 287-290, 2014.

LAPRIDA, C., B. VALERO GARCÉS. Cambios ambientales de épocas históricas en la pampa bonaerense en base a ostrácodos: historia hidrológica de la laguna de Chascomús. Ameghiniana v. 46 n. 1, p. 95-111, 2009.

MIRAGLIA, M. Los Relatos de Viajeros y Científicos como Fuente Documental para la Reconstrucción Histórica del Territorio de las Cuencas del Reconquista y Las Encadenadas en la Provincia de Buenos Aires (Argentina), Siglo XIX. Fronteiras: Journal of Social, Technological and Environmental Science v.6, n.1, p. 183-197, 2017.

MIRAGLIA, M. La Geografía, la Historia y las aplicaciones de las TIGs en los estudios territoriales multitemporales. Párrafos Geográficos v. 15, n. 1, p. 65-90, 2016. 
PARDIÑAS, U.F.J., G.J. MOREIRA, C.M. GARCÍA ESPONDA Y L.J.M. DE SANTIS. Deterioro ambiental y micromamíferos durante el Holoceno en el nordeste de la estepa patagónica (Argentina). Revista Chilena de Historia Natural v. 73, n. 1, p. 9-21, 2000.

PRIETO, M.R., P.E. VILLAGRA, N.B. LANA y E.M Abraham. Utilización de documentos históricos en la reconstrucción de la vegetación de la Llanura de la Travesía (Argentina) a principios del siglo XIX. Revista Chilena de Historia Natural v. 76, n. 4, p. 613-622, 2003.

ROJAS, J.F., M.R. PRIETO, J. ÁLVAREZ Y E. CESCA. Procesos socioeconómicos y territoriales en el uso de los recursos forestales en Mendoza desde fines de siglo XIX hasta mediados del XX. Desarrollo y Territorio v. 2, n. 7, 2009.

SABBATELLA, I. Apuntes para una historia ecológico-política de la explotación de los hidrocarburos en Argentina. X Jornadas de Sociología. Facultad de Ciencias Sociales, Universidad de Buenos Aires, Buenos Aires, 2013.

SALDI, L. Y L. WAGNER. Aportes antropológicos a la Historia Ambiental en contextos y estudios latinoamericanos. Revista Latino-Americana de História v. 2, n. 8, p. 8-30, 2013.

STUTZ, S., M.S. TONELLO, M. A. GONZÁLEZ SAGRARIO, D. NAVARRO Y S. L. FONTANA. Historia ambiental de los lagos someros de la llanura Pampeana (Argentina) desde el Holoceno medio: inferencias paleoclimáticas. Latin American Journal of Sedimentology and Basin Analysis v. 21 n. 2, p. 119-138, 2014.

TORRELLA, S.A. y J. ADÁMOLI. Situación ambiental de la Ecorregión del Chaco seco, in: La Situación Ambiental Argentina 2005, A. Brown, U. Martinez Ortiz, M. Acerbi y J. Corcuera Editores, Buenos Aires: Ed. Fundación Vida Silvestre Argentina, 2006, p. 75-82.

TORRES, L. M. Hilos de agua, lazos de sangre: enfrentando la escasez en el desierto de Lavalle (Mendoza, Argentina). Ecosistemas v. 17, n. 1, p. 46-59, 2008.

URQUIZA, S.V., A. S. ROMANO y S. M. L. LÓPEZ CAMPENY. Espacios recurrientes... Historias diferentes: Una mirada desde la arqueofauna. Sitio Piedra Horadada 2, Antofagasta de La Sierra, Catamarca, Argentina, in: IZETA, A., y GOÑALONS, G. L. M. (Eds), De la Puna a las Sierras: Avances y Perspectivas en Zooarqueología Andina. Oxford: Archaeopress, 2013.

VARELA, B. y C. CARBALLO. Recursos, sociedade y ambiente: el ciclo del oro en la organización ambiental del território serrano de la Provincia de San Luis del siglo XVIII (Argentina). Estudos Ibero-Americanos, v. 27, n. 2, p. 31-47, 2001.

ZARRILLI, A. Argentina, tierra de promisión. Una interpretación historiográfica de las relaciones entre la historia rural y la historia ambiental. Revista de Historia Iberoamericana, v. 7, n. 1, p. 107-132, 2014.

ZARRILLI, A.G. y A. SALOMÓN (comp). Historia, política y gestión ambiental: perspectiva y debates. Editora Imago Mundi, 2012.

ZARRILLI, A. Transformación ecológica y precariedad económica en una economía marginal. El Gran Chaco argentino, 1890-1950. Revista Theomai, n. 1, 2000. 